\title{
The super-soft source XMMU J052016.0-692505 in the LMC ${ }^{\star}$
}

\section{A likely white dwarf $\mathrm{Be} / \mathrm{X}$-ray binary}

\author{
P. Kahabka ${ }^{1}$, F. Haberl ${ }^{1}$, J. L. Payne ${ }^{2}$, and M. D. Filipović ${ }^{3}$ \\ 1 Max-Planck-Institut für extraterrestrische Physik, Giessenbachstrasse, 85741 Garching, Germany \\ e-mail: [pkahabka; fwh] @mpe.mpg.de \\ 2 Centre for Astronomy, James Cook University, Townsville, Queensland 4811, Australia \\ 3 University of Western Sydney, Locked Bag 1797, Penrith South DC, NSW 1797, Australia
}

Received 25 April 2006 / Accepted 28 July 2006

\section{ABSTRACT}

\begin{abstract}
Aims. We report the discovery of the super-soft X-ray source XMMU J052016.0-692505 in the LMC with XMM-Newton. Methods. We analyse the EPIC spectra of XMMU J052016.0-692505 and study the likely optical counterpart LMCV2135.

Results. Using an absorbed blackbody spectrum we derive a bolometric luminosity of $\gtrsim 10^{34} \mathrm{erg} \mathrm{s}^{-1}$ for the X-ray source at LMC distance. Assuming that the bolometric luminosity does not exceed the Eddington luminosity of a $\sim 1.0 M_{\odot}$ star we derive a blackbody temperature of (25-70) eV and an LMC absorbing column density of $<6 \times 10^{21} \mathrm{~cm}^{-2}$. The likely optical counterpart of XMMU J052016.0-692505 is the LMC variable star LMCV2135 which is a MACHO and OGLE variable. The infrared and optical colors and magnitudes of this star are consistent with a hot star of likely spectral type B. The long-term MACHO light curve shows variability with a timescale of $\sim 500$ and $\sim 1000$ days. The optical spectra obtained at the 1.9-meter telescope of the South African Astronomical Observatory show strong $\mathrm{H}_{\alpha}$ and $\mathrm{H}_{\beta}$ emission lines (with $E W_{\mathrm{H}_{\alpha}} \sim 34 \AA$ ) which indicate a B0-3e star. The radial velocities of the $\mathrm{H}_{\alpha}$ and $\mathrm{H}_{\beta}$ emission lines show a variation from $\sim 400-450 \mathrm{~km} \mathrm{~s}^{-1}$ to $\sim 5-20 \mathrm{~km} \mathrm{~s}{ }^{-1}$ which is consistent with the systemic velocity of the LMC and an intrinsic variation most likely due to the rotation of the Be disk. We discuss LMCV2135/XMMU J052016.0-692505 as a Be/white dwarf binary system in the LMC. The super-soft X-ray spectrum of the source could be due to a stable nuclear burning white dwarf with a mass of $\sim 0.9-1.0 M_{\odot}$.
\end{abstract}

Key words. Magellanic Clouds - X-rays: stars - X-rays: binaries - stars: emission-line, Be - stars: general

\section{Introduction}

Super-soft X-ray sources are characterized by very soft spectra with counts detected mainly below $\sim 0.5-1.0 \mathrm{keV}$. They were observed in the Magellanic Clouds, the Galaxy and in more than 10 external galaxies (cf. Kahabka \& van den Heuvel 2006 for a recent review). Many of the super-soft sources in the Magellanic Clouds and the Galaxy were optically identified with either close (orbital periods $\sim 9 \mathrm{~h}$ to $4 \mathrm{~d}$ ) or wide (orbital periods $\sim 300-1600$ days) binaries. Several of the super-soft sources are identified with the post-nova phase of a classical (orbital periods $\sim 85 \mathrm{~m}$ to $3.5 \mathrm{~h}$ ), recurrent, or symbiotic nova. Many observed characteristics of binary super-soft sources can be explained by steady or stable nuclear burning of H-rich matter accreted onto $\mathrm{CO}$ (or ONeMg) white dwarfs (WDs). Effective blackbody temperatures of $\sim 20-100 \mathrm{eV}$ and (bolometric) luminosities of $\sim 10^{36}-10^{38} \mathrm{erg} \mathrm{s}^{-1}$ were inferred from X-ray spectra, characteristic of steady nuclear burning on $\sim 0.6-1.4 M_{\odot}$ WDs (see van den Heuvel et al. 1992; Nomoto et al. 2006). Accretion rates required for steady nuclear burning (on "cold" WDs) are $\sim(1-6) \times 10^{-7} M_{\odot} \mathrm{yr}^{-1}$. It was proposed that hot WD models require lower accretion rates (Starrfield et al. 2004; see also Nomoto et al. 2006).

* Based on observations with XMM-Newton, an ESA Science Mission with instruments and contributions directly funded by ESA Member states and the USA (NASA).
Studying individual binary super-soft sources may allow to derive constraints on the mass of the WD and the donor star, derive the orbital period and constrain the accretion rate. In many systems the super-soft phase is observed to be short (from weeks to years) and several scenarii can account for a short duration of a super-soft phase: a variable accretion rate giving rise to envelope oscillations, varying system intrinsic obscuration effects or a limited reservoir of nuclear fuel for post-nova systems. Besides binary super-soft sources single star super-soft sources like hot pre-WDs (PG1159 stars) and hot nuclei of planetary nebulae were observed. Also cooling neutron stars (NSs) and magnetic cataclysmic variables (polars) can have a super-soft component but of low luminosity.

A program was initiated to observe and study faint ROSAT discovered super-soft sources in the fields of the Magellanic Clouds with XMM-Newton (cf. Kahabka \& Haberl 2006 for candidate super-soft sources in the field of the Small Magellanic Cloud). This work is complemented by a search for new supersoft sources in archival XMM-Newton observations of the LMC field. Here we report on the discovery, optical identification, and study of a new super-soft source XMMU J052016.0-692505 in the LMC.

\section{XMM-Newton observations}

XMMU J052016.0-692505 was discovered in a search for new super-soft X-ray sources in the EPIC-pn data from the public 
Table 1. XMM-Newton EPIC observation of XMMU J052016.0-692505.

\begin{tabular}{llllcccc}
\hline \hline Target & Instrument & $\begin{array}{c}\text { Read-out } \\
\text { Mode }\end{array}$ & Filter & $\begin{array}{c}\text { Sat. Revol./ } \\
\text { Obs.-ID }\end{array}$ & Start & Observation & $\begin{array}{c}\text { Exp. } \\
\text { End }\end{array}$ \\
\hline SNR 0520-69.4 & EPIC-pn & FF, 73 ms & Medium & $\begin{array}{c}752 \\
\text { [ks }\end{array}$ & Jan. 17, 2004, 21:16 & Jan. 18, 2004, 05:43 & 30.4 \\
& EPIC-MOS1/2 & FF, 2.6 s & Medium & & Jan. 17, 2004, 20:54 & Jan. 18, 2004, 03:32 & 23.6 \\
\hline
\end{tabular}

$X M M-N e w t o n$ archive in the direction towards the LMC. XMMU J052016.0-692505 was detected in an EPIC-pn observation performed from 17 to 18 January 2004 and pointed at the LMC supernova remnant SNR 0520-69.4.

The data were collected with the European Photon Imaging EPIC cameras with the $p n$ CCD detector (Strüder et al. 2001) and the MOS1 and MOS2 CCDs (Turner et al. 2001) behind the three X-ray telescopes (Aschenbach et al. 2000). The observation is summarized in Table 1.

We created the EPIC event files using "epchain" with the parameter setting screenlowthresh $=0$ and "emchain" with default setting. For the extraction of the spectrum we used only single pixel events for $p n$ and the setting PATTERN $\leq 12$ for $M O S$. For spectral fitting we calculated effective areas and redistribution matrices for EPIC-pn and EPIC-MOS using SAS 6.5 tools, but we included in the spectral analysis only events above $170 \mathrm{eV}$ as source counts were observed mainly above $200 \mathrm{eV}$. We performed spectral fits using XSPEC v11.3.2. Source net count rates were derived for EPIC-pn from the energy spectra in the given energy bands. Optical monitor $(O M)$ data (Mason et al. 2001) were used to determine the count rate and flux in the optical. The X-ray position of XMMU J052016.0-692505 as determined from a maximum likelihood fit is RA $(\mathrm{J} 2000)=$ $5^{\mathrm{h}} 20^{\mathrm{m}} 16^{\mathrm{s}} .0, \operatorname{Dec}(\mathrm{J} 2000)=-69^{\circ} 25^{\prime} 5^{\prime \prime} .8$.

\subsection{EPIC spectral analysis}

The source was observed with the EPIC-pn detectors with a net exposure time of $20.7 \mathrm{ks}$ and a count rate of $(2.40 \pm$ $0.64) \times 10^{-3} \mathrm{~s}^{-1}(0.2-1.0 \mathrm{keV})$. Source counts are detected below $1 \mathrm{keV}$ in agreement with a super-soft source. We extracted the source counts in a $15^{\prime \prime}$ circle and the background counts from a nearby $25^{\prime \prime}$ circle and collected $\sim 50$ source counts for X-ray spectral fitting.

XMMU J052016.0-692505 was also detected with the EPIC-MOS detectors with a net exposure of $22.2 \mathrm{ksec}$ and a count rate of $(1.48 \pm 0.51) \times 10^{-3} \mathrm{~s}^{-1}$. We extracted the source counts in an $25^{\prime \prime}$ circle and the background counts from a nearby $25^{\prime \prime}$ circle and collected $\sim 33$ source counts for X-ray spectral fitting.

We used a blackbody spectral model modified by galactic absorption using the solar elemental abundances of Anders \& Grevesse (1989) and in addition by absorption due to LMC gas with an abundance of 0.5 solar. In the direction of XMMU J052016.0-692505 the galactic foreground $N_{\mathrm{H}}$ is $4.7 \times 10^{20} \mathrm{~cm}^{-2}$ which we used as fixed value in the fit. The total LMC $N_{\mathrm{H}}$ is $1.1 \times 10^{21} \mathrm{~cm}^{-2}$ (Brüns et al. 2005). A simultaneous fit with all model parameters the same for $p n$ and $M O S$ spectra was performed using the blackbody model (Fig. 1). The results are summarized in Table 2.

The $90 \%$ confidence errors have been determined from the LMC $N_{\mathrm{H}}$ - blackbody $k T$ chi-squared plane using the constraint that the luminosity does not exceed the Eddington luminosity of a $1 M_{\odot}$ star of $\sim 10^{38} \mathrm{erg} \mathrm{s}^{-1}$ (Fig. 2). We used the C-statistics

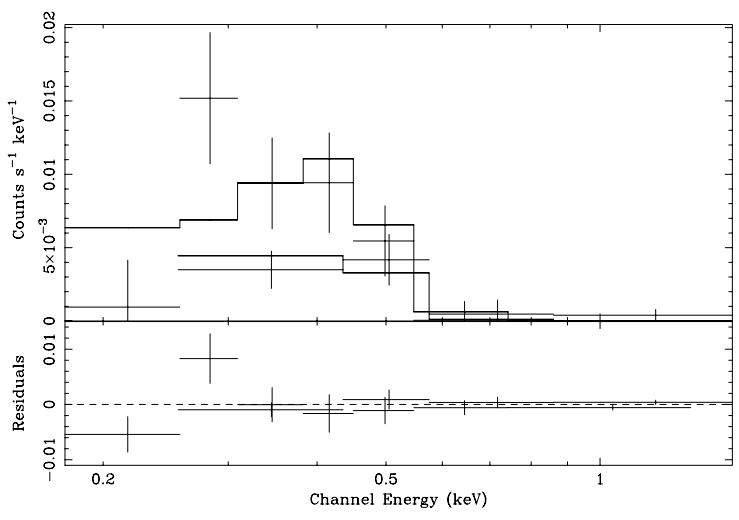

Fig. 1. EPIC-pn (thick line) and combined MOS1 and MOS2 (thin line) spectrum of XMMU J052016.0-692505 together with the bestfit blackbody model.

(Cash 1979) in XSPEC as there are only 2-17 background subtracted source counts per spectral bin from the $p n$ and 3-9 from the MOS.

\subsection{EPIC-pn light curves}

We generated EPIC-pn light curves of XMMU J052016.0692505 using a binsize of 1 hour in the "soft" $(0.17-0.4 \mathrm{keV})$ and the "hard" $(0.4-0.7 \mathrm{keV})$ band. There is some variation seen in the soft count rate during the observation (although the uncertainties are large). The statistics is insufficient to reveal any significant intensity or hardness ratio variations.

\section{ROSAT observations}

XMMU J052016.0-692505 was observed but not detected with the inner part $\left(\leq 20^{\prime}\right)$ of the ROSAT PSPC detector during a few observations with a short exposure time of $\sim 140-240 \mathrm{~s}$. XMMU J052016.0-692505 was also observed with the ROSAT HRI during a $18 \mathrm{ksec}$ observation performed from July 11 to October 12, 1997. We applied a maximum likelihood detection to the data and found a source with a likelihood ratio of 6.3 (which equals a probability of a spurious detection of $0.2 \%)$ at the position RA $(\mathrm{J} 2000)=5^{\mathrm{h}} 20^{\mathrm{m}} 16^{\mathrm{s}} .9$, Dec $(\mathrm{J} 2000)=-69^{\circ} 25^{\prime} 9^{\prime \prime}$ ( $3^{\prime \prime} 90 \%$ confidence error radius), which agrees within the statistical and systematic error due to the attitude with the position of XMMU J052016.0-692505. We derive a HRI count rate of $(5.1 \pm 2.2) \times 10^{-4} \mathrm{~s}^{-1}$ which corresponds only to a $2.3 \sigma$ detection.

\section{Optical identification}

The EPIC-pn position of XMMU J052016.0-692505 is consistent with the position of the LMC variable star LMCV2135 at a distance of $0 .^{\prime} 4$ from the optical position $\left(\mathrm{RA}(\mathrm{J} 2000)=05^{\mathrm{h}} 20^{\mathrm{m}} 16^{\mathrm{s}} .08\right.$ and $\left.\operatorname{Dec}(\mathrm{J} 2000)=-69^{\circ} 25^{\prime} 5^{\prime \prime} .8\right)$. 
Table 2. XMM-Newton EPIC-pn+MOS spectral fit results for XMMU J052016.0-692505. Galactic absorbing column $N_{\mathrm{H}}^{\mathrm{gal}}$, LMC absorbing column $N_{\mathrm{H}}^{\mathrm{LMC}}$ and blackbody temperature $k T_{\mathrm{bb}}$, constrained assuming the bolometric luminosity does not exceed the Eddington luminosity of a $1.0 M_{\odot}$ star of $\sim 10^{38} \mathrm{erg} \mathrm{s}^{-1}$, the absorbed flux flux ${ }^{\text {abs }}$ and unabsorbed flux flux ${ }^{\text {unabs }}$ and luminosity $L^{\text {unabs }}$, for $0.1-2.4 \mathrm{keV}$. The C statistics is 11.5 for 15 phase bins. Also given are the reduced chi-squared and the degrees of freedom for the same best-fit parameters but using the chi-squared statistics.

\begin{tabular}{lcccccccc}
\hline \hline Instrument & $\begin{array}{c}N_{\mathrm{H}}^{\text {gal }} \\
\left(10^{20} \mathrm{~cm}^{-2}\right)\end{array}$ & $\begin{array}{c}N_{\mathrm{H}}^{\mathrm{LMC}} \\
\left(10^{20} \mathrm{~cm}^{-2}\right)\end{array}$ & $\begin{array}{c}k T_{\mathrm{bb}} \\
(\mathrm{eV})\end{array}$ & $\begin{array}{l}\text { flux } \\
\left(10^{-13} \mathrm{erg} \mathrm{cm}^{-2} \mathrm{~s}^{-1}\right)\end{array}$ & $\begin{array}{c}L^{\text {unabs }} \\
\left(10^{36} \mathrm{erg} \mathrm{s}^{-1}\right)\end{array}$ & $\chi_{\text {red }}^{2}$ & d.o.f. \\
\hline EPIC-pn+MOS & 4.7 (fixed) & $28_{-28}^{+30}$ & $33_{-8}^{+34}$ & 0.055 & 184 & 5.5 & 0.91 & 12 \\
\hline
\end{tabular}

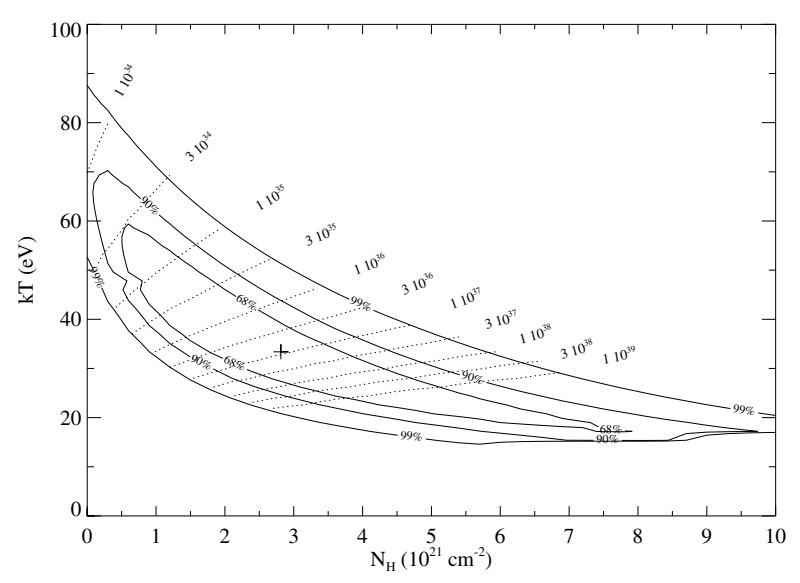

Fig. 2. $68 \%, 90 \%$, and $99 \%$ confidence $L M C N_{\mathrm{H}}-k T$ contours of XMMU J052016.0-692505 for the EPIC-pn+MOS data. The best-fit is given with $\mathrm{a}+$. Dashed lines give the bolometric luminosity from $10^{34}$ to $10^{39} \mathrm{erg} \mathrm{s}^{-1}$.

The star is classified as a poorly studied optical variable (Samus 2004).

\subsection{XMM-Newton OM observation}

LMCV2135 was also in the field of view of the OM. To verify the optical counterpart, which we found by correlation with catalogues in VizieR, we adjusted the OM UVM2 image using objects from the Massey (2002) UBVR CCD survey catalogue of the Magellanic Clouds. We derived a shift of -0.5 in right ascension and $+1^{\prime \prime}$.7 in declination, which we applied to the $O M$ image, Fig. 3. We clearly find a star at the position of the X-ray source. To estimate the optical $V$ magnitude of the optical star, we derived count rates of $12.92 \pm 0.06 \mathrm{~s}^{-1}$ in the UVM2 (4260 s exposure) and of $30.54 \pm 0.08 \mathrm{~s}^{-1}$ in the UVW1 (5000 s exposure) filter. Assuming a B0 spectral type we derive a $V$ magnitude of $15.45 \pm 0.05$ from both filter measurements, correcting for deadtime and coincidence losses (Sect. 3.5.6 of $X M M-N e w t o n$ User's Handbook). We note here, that the $O M$ derived $V$ magnitude strongly depends on the assumed spectral type. We derive from the count rates in the two filters fluxes of $2.84 \times 10^{-14} \mathrm{erg} \mathrm{cm}^{-2} \mathrm{~s}^{-1}$ and $1.45 \times 10^{-14} \mathrm{erg} \mathrm{cm}^{-2} \mathrm{~s}^{-1}$ at $2310 \AA$ and $2910 \AA$ A respectively.

\subsection{MACHO and OGLE light curves}

LMCV2135 is identical to the MACHO star 78.6466.23 and the $O G L E$ variable OGLE 052016.25-692505.3 (Udalski et al. $1997,1998)$ with $B=14.77 \pm 0.01, V=14.80 \pm 0.02$, and $I=14.68 \pm 0.04 \mathrm{mag}$ and the $B-V=-0.03 \pm 0.03$ and $V-I=0.12 \pm 0.04$ (cf. Fig. 4). These colors and magnitudes

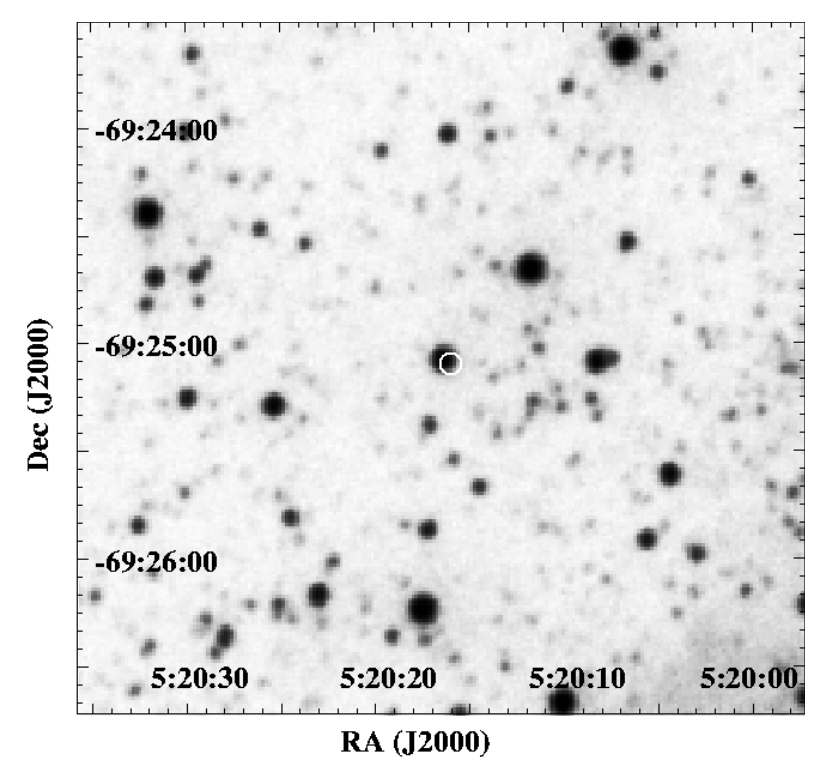

Fig. 3. $O M$ filter UVM2 image centered on the position of XMMU J052016.0-692505. The image has been adjusted to stars from the Massey (2002) catalog. The X-ray position of XMMU J052016.0-692505 is given with a circle with radius 3".

are consistent with a Be star or Be/X-ray binary in the LMC (Mennickent et al. 2002). We note that the $M A C H O$ calibrated $B$ mag derived with the Web site display differs only by $\sim 0.2 \mathrm{mag}$ from our light curve adjusted to the OGLE B-mag light curve but it is systematically by +0.75 mag too bright (see Schmidtke \& Cowley 2005).

An FFT analysis has been applied to the MACHO $R$ and $B$ band and the OGLE I-band data (Lomb 1976; Scargle 1982). The MACHO light curves were retrieved with the interactive light curve browser ${ }^{1}$. High power $(>40)$ was found for periods of $510 \pm 20$ days in the $R$ and $B$-band and $\sim 1040 \pm 70$ days in the $R$-band data (Fig. 5). There is also a long-term trend in the $M A C H O R$ and $B$-band data with increasing intensity over the 2500 days of observations. We cover only a few cycles for both periodicities in the $\mathrm{MACHO}$ observation and cannot judge on a periodic nature.

\subsection{Optical spectroscopy}

Spectral observations of LMCV2135 were conducted between 23 and 30 January, 2006 using the 1.9-m telescope and Cassegrain spectrograph at the South African Astronomical Observatory (SAAO) in Sutherland. A total of 7 spectra were obtained using grating number 7 (300 lines $\left.\mathrm{mm}^{-1}\right)$ between 4000 and $7000 \AA$ with a spectral resolution of $5 \AA$.

\footnotetext{
${ }^{1}$ http://www .macho.mcmaster.ca/Data/MachoData.html
} 


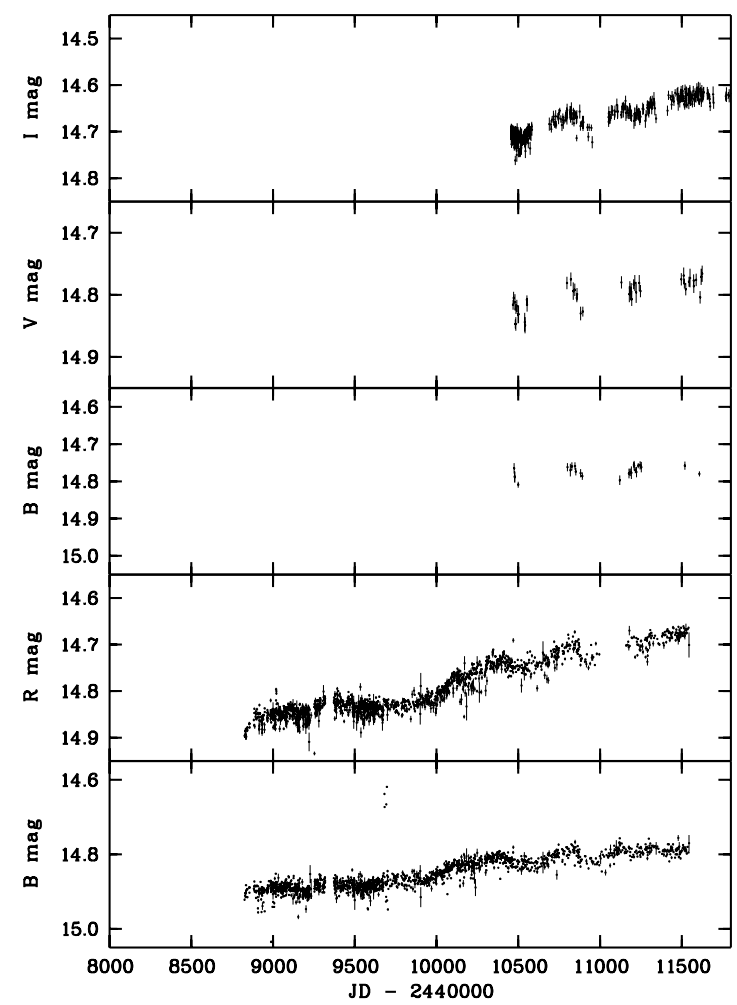

Fig. 4. $O G L E I I$ calibrated $I, V$ and $B$-band (first three panels), and $M A C H O R$ and $B$-band light curve (uncalibrated light curve, adjusted to the OGLE II B-band light curve, lower two panels) of XMMU J052016.0-692505.

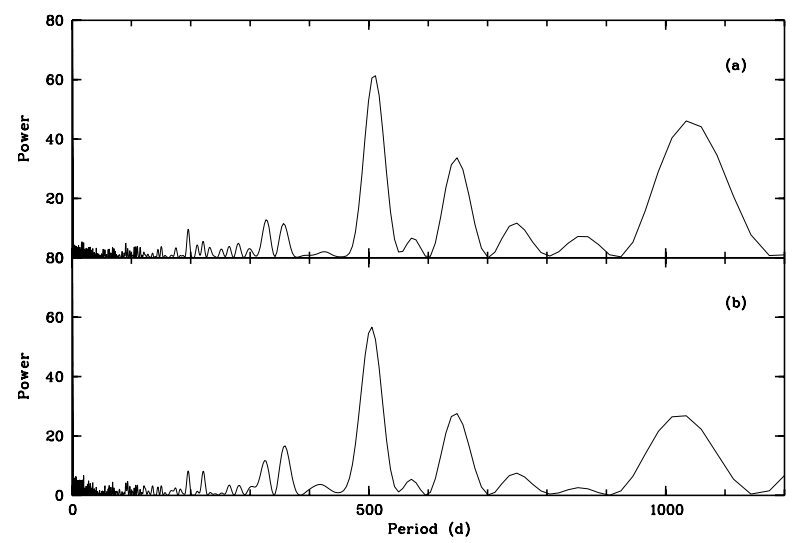

Fig. 5. FFT of the red a) and blue b) $M A C H O$ light curve of LMCV2135.

The spectra were calibrated and cleaned using standard routines provided in the IRAF and FIGARO software package. After extraction and wavelength calibration, the one dimensional spectra were flux calibrated using the spectrophotometric standard EG 21. We estimate that these fluxes have up to 50 percent variation from night to night as the spectra were obtained during an independent project to study supernova remnants and superbubble shocks and flux ratios rather than absolute fluxes were of interest. These fluxes therefore should not be considered for absolute spectrophotometry. However, comparison of radial velocities and relative fluxes of emission lines are reliable. An example spectrum is shown in Fig. 6. The $\mathrm{H}_{\alpha}$ and $\mathrm{H}_{\beta}$ emission lines were analyzed for position, equivalent width, Gaussian full width at half maximum (FWHM) and flux using "splot" found in the IRAF software suite. Radial velocities were determined using

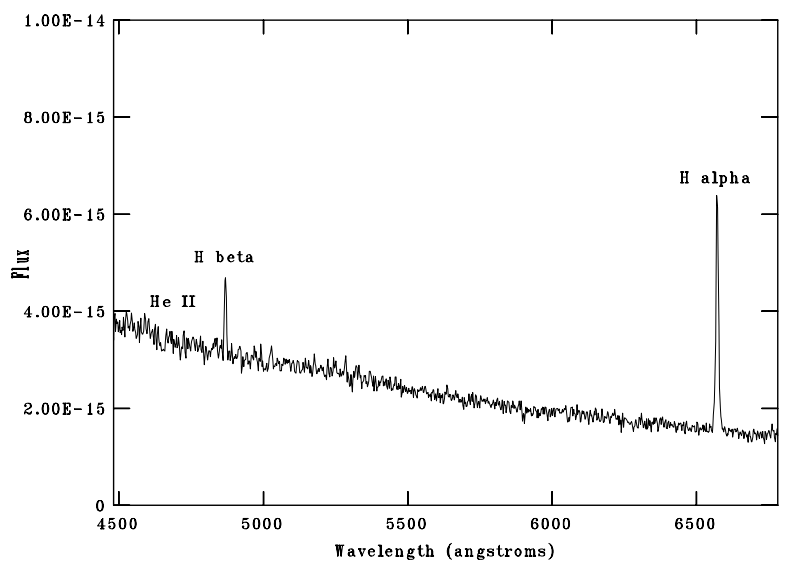

Fig. 6. Optical flux spectrum (erg $\left.\mathrm{cm}^{-2} \mathrm{~s}^{-1} \AA^{-1}\right)$ of XMMU J052016.0-692505 for day JD 2453759.36 (cf. Table 3 for the observation parameters). The positions of the He II ( $\lambda$ 4686), $\mathrm{H}_{\beta}$ $(\lambda$ 4861) and $\mathrm{H} \alpha(\lambda$ 6563) lines are shown.

emsao from IRAF's rvsao package. The observations and results are summarized in Table 3.

\section{Discussion}

The X-ray luminosity of $10^{34}$ to $10^{38} \mathrm{erg} \mathrm{s}^{-1}$ of XMMU J052016.0-692505 inferred from the EPIC data is consistent with luminosities of Be/NS X-ray binaries. But the Xray spectrum is much softer than observed in $\mathrm{Be} / \mathrm{NS}$ binaries. The luminosity is above the luminosity of accreting WDs but in the range of stable nuclear burning WDs. Thus XMMU J052016.0-692505 could be the first Be/X-ray binary with a WD as compact object where the WD exhibits super-soft $\mathrm{X}$-ray emission due to stable nuclear burning. Only a few other $\mathrm{Be} / \mathrm{X}$-ray binaries including $\gamma$ Cas were proposed to harbour a WD due to their low X-ray luminosity (Oliveira et al. 2006). But in these cases the X-ray spectrum is hard and very similar to that observed from Be/X-ray binaries with a NS. Thus if the super-soft X-ray spectrum observed with EPIC is due to a steadily nuclear burning WD, then XMMU J052016.0-692505 could be a Be/WD X-ray binary in the LMC. A spectral type of $\mathrm{B} 0-3 \mathrm{e}$ would be consistent with the range of spectral types predicted from population synthesis calculations for Be/WD binaries (Pols et al. 1991).

\subsection{The Be star and disk}

The optical continuum (e.g. the observation of JD 2453761.42 ) can be reproduced with a synthetic stellar spectrum (Lejeune et al. 1997) with a temperature of $1.6 \times 10^{4} \mathrm{~K}$ (metallicity 0.5 solar) and a stellar radius of $10.9 R_{\odot}$, consistent with a $\sim 8 M_{\odot}$ star (giant, luminosity class III) (Fig. 7).

A visual extinction $A_{\mathrm{V}}=0.81$ has been used. Depending on the assumed optical extinction and stellar radius the observed optical continuum is consistent with a range of stellar temperatures of $\sim 12000-30000 \mathrm{~K}$. Most models are consistent with the evolutionary track of a $\sim(7-16) M_{\odot}$ star, equivalent to a spectral type B0-3 (Harmanec 1988). We note that although the fluxes of our optical spectra may not be better determined than $\sim 50 \%$ they still can be used to qualitatively estimate the stellar parameters. But we cannot infer a unique solution of stellar parameters.

We may further constrain the stellar parameters by taking into account the fluxes measured in the UV with the $O M$ filters 
Table 3. Optical spectroscopy of LMCV2135. The date of the observation (JD), exposure time, flux (10 $\left.0^{-14} \mathrm{erg} \mathrm{cm}^{-2} \mathrm{~s}^{-1}\right)$, equivalent width (EW), radial velocity (RV), and FWHM of the $\mathrm{H}_{\alpha}$ and $\mathrm{H}_{\beta}$ emission line is given. The mean $90 \%$ confidence errors of the $\mathrm{H}_{\alpha}$ and $\mathrm{H}_{\beta} E W$ are 3.7 and $2.0 \AA$, of the $\mathrm{H}_{\alpha}$ and $\mathrm{H}_{\beta} F W H M, 1.1$ and $3.9 \AA$ respectively.

\begin{tabular}{rccccccccccc}
\hline \hline Date & \multirow{2}{*}{$\begin{array}{c}\text { Exp } \\
(\mathrm{s})\end{array}$} & \multicolumn{2}{c}{ Flux } & \multicolumn{2}{c}{ ratio } & \multicolumn{2}{c}{$E W$} & \multicolumn{2}{c}{ Radial velocity } & \multicolumn{2}{c}{$F W H M$} \\
& & & & $\mathrm{H}_{\beta}$ & $\frac{\mathrm{H}_{\alpha}}{\mathrm{H}_{\beta}}$ & $\begin{array}{c}\mathrm{H}_{\alpha} \\
(\AA)\end{array}$ & $\begin{array}{c}\mathrm{H}_{\beta} \\
(\AA)\end{array}$ & $\begin{array}{c}\mathrm{H}_{\alpha} \\
\left(\mathrm{km} \mathrm{s}^{-1}\right)\end{array}$ & $\begin{array}{c}\mathrm{H}_{\beta} \\
\left(\mathrm{km} \mathrm{s}^{-1}\right)\end{array}$ & $\begin{array}{c}\mathrm{H}_{\alpha} \\
(\AA)\end{array}$ & $\begin{array}{c}\mathrm{H}_{\beta} \\
(\AA)\end{array}$ \\
\hline 2453759.36 & 1200 & 5.37 & 1.33 & 4.04 & -34.8 & -4.2 & 433.5 & 450.8 & 10.4 & 7.4 \\
61.42 & 1200 & 7.09 & 1.57 & 4.52 & -35.6 & -4.0 & 295.6 & 267.7 & 9.5 & 7.9 \\
62.48 & 800 & 11.2 & 2 & 5.6 & -34.1 & -3.9 & 333.9 & 277.3 & 9.7 & 8.2 \\
63.41 & 1200 & 5.28 & 0.84 & 6.27 & -33.4 & -4.3 & 280.6 & 264.2 & 9.8 & 7.8 \\
64.47 & 800 & 4.3 & 0.82 & 5.24 & -31.7 & -3.8 & 95.0 & 18.1 & 9.8 & 5.3 \\
65.58 & 800 & 4.17 & - & - & -37.3 & - & 5.9 & - & 9 & - \\
66.32 & 1200 & 7.66 & 1.8 & 4.32 & -33.5 & -4.1 & 326.5 & 321.9 & 9.9 & 7.5 \\
\hline
\end{tabular}
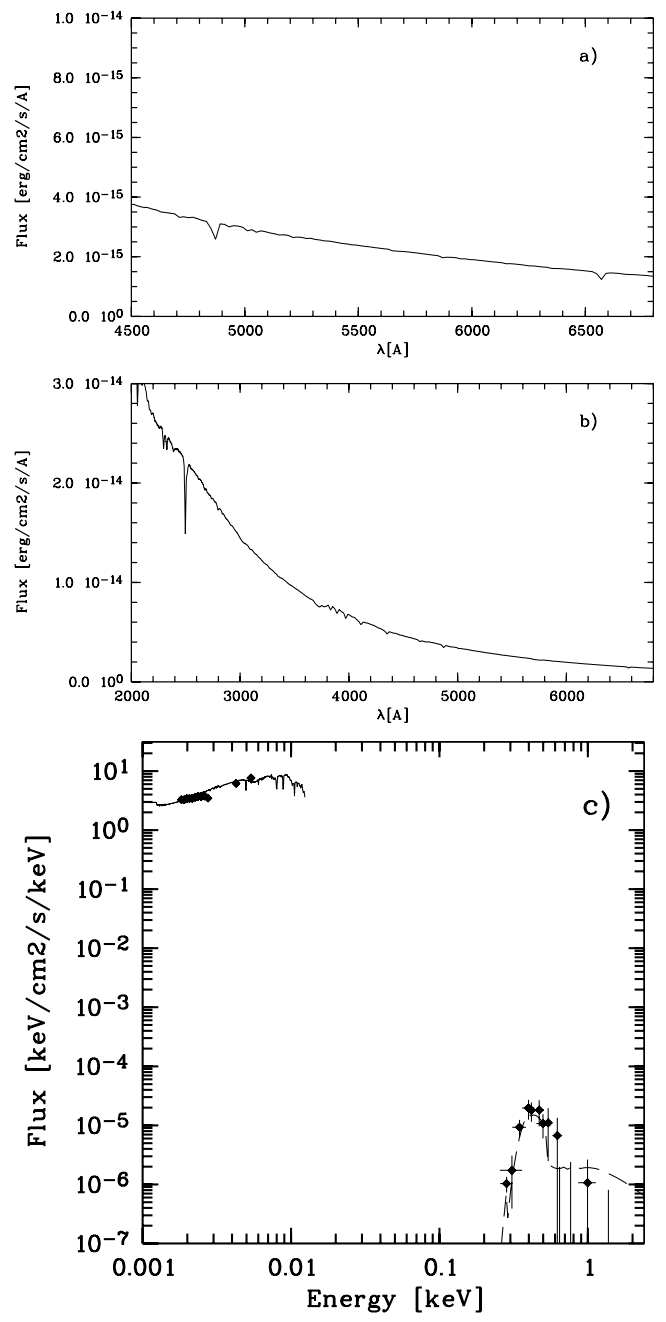

Fig. 7. a) Synthetic flux spectrum of a B star $\left(T_{\text {eff }}=16000 \mathrm{~K}, \log g=\right.$ $\left.3.5, R=10.9 R_{\odot}\right)$ with $A_{\mathrm{V}}=0.76,\left(N_{\mathrm{H}}=1.2 \times 10^{21} \mathrm{~cm}^{-2}\right)$ which reproduces the optical spectrum of XMMU J052016.0-692505 measured on day JD 2453759.36 (cf. Fig. 6). $\mathrm{H}_{\alpha}$ and $\mathrm{H}_{\beta}$ are seen in absorption from the stellar spectrum as we do not include the contribution from the Be disk. b) Synthetic flux spectrum of a B star $\left(T_{\text {eff }}=30000 \mathrm{~K}\right.$, $\left.\log g=3.5, R=5.1 R_{\odot}\right)$ with $A_{\mathrm{V}}=0.33,\left(N_{\mathrm{H}}=5.0 \times 10^{20} \mathrm{~cm}^{-2}\right)$ which reproduces the optical spectrum of XMMU J052016.0-692505 measured on day JD 2453759.36 (cf. Fig. 6) and the OM UV measurements. $\mathrm{H}_{\alpha}$ and $\mathrm{H}_{\beta}$ are seen in absorption from the stellar spectrum. We include in addition a powerlaw continuum for the Be disk $\left(F_{\lambda}=2.0 \times 10^{-6} \lambda^{-3}\right.$, e.g. Hony et al. 2000). c) X-ray, UV and optical model spectra (from $b$ ) and observational data points.
UVW1 and UVM2. Consideration of the UV measurements requires a $\sim 30000 \mathrm{~K}$ stellar spectrum (metallicity 0.3 solar) and a stellar radius of $5.1 R_{\odot}$, consistent with a $\sim 15 M_{\odot}$ star of spectral type B0 (main-sequence, luminosity class $\mathrm{V}$ ). The $V$-magnitude for such a spectrum is $\sim 15.6 \pm 0.2$ and consistent with the magnitude estimated in Sec. 4.1. We note that from population synthesis calculations it follows that a Be star with a mass of $\sim 14 M_{\odot}$ and a WD with a mass of $\sim 1.2 M_{\odot}$ are the most likely to be observed (Pols et al. 1991). Such masses are reasonably close to the masses inferred from our XMM-Newton and optical data.

In addition strong $\mathrm{H}_{\alpha} \lambda 6563$ and (with the exception of the observation JD 2453765.58) $\mathrm{H}_{\beta} \lambda 4861$ emission lines are observed. We do not detect the HeII $\lambda 4686$ line which is consistent with the emission lines arising in the Be disk. The $\mathrm{H}_{\alpha}$ lines have an equivalent width $E W_{\mathrm{H}_{\alpha}} \sim 32-37 \AA$ and a mean full-width at half maximum $F W H M \sim 8.3 \pm 1.1 \AA$ (taking into account an instrumental width of $5 \AA$ ), which corresponds to $\sigma_{\mathrm{H}_{\alpha}}=F W H M_{\mathrm{H}_{\alpha}} / 2.35=3.5 \AA$ and a velocity of $\sim 160 \mathrm{~km} \mathrm{~s}^{-1}$. The large observed equivalent width of the $\mathrm{H}_{\alpha}$ line may be explained by a large radius of the equatorial disk of the Be star and a high rotational velocity of the star, i.e. a large value of $v \sin i$. Indeed an $\mathrm{H}_{\alpha}$ equivalent width of $32-37 \AA$ and a rotational velocity of $160 \mathrm{~km} \mathrm{~s}^{-1}$ are consistent with the maximum equivalent width of stars of spectral type B0-3e. A higher value of $v \sin i=293 \mathrm{~km} \mathrm{~s}^{-1}$ is from Eq. (7) of Dachs et al. (1986). Such a velocity would be below the escape velocity of $500 \mathrm{~km} \mathrm{~s}^{-1}$ of a $8 M_{\odot}, R=11 R_{\odot}$ star and of $1000 \mathrm{~km} \mathrm{~s}^{-1}$ of a $15 M_{\odot}, R=5 R_{\odot}$ star respectively. The $\mathrm{H}_{\beta}$ line is expected to form in the innermost regions of the stellar disk. The stronger variability of the $\mathrm{H}_{\beta}$ flux (with the disappearance during one observation) may indicate that there are large variations in the mass flow in the inner disk. We note that a rotational velocity of $160 \mathrm{~km} \mathrm{~s}^{-1}$ and a stellar radius of $11 R_{\odot}\left(5 R_{\odot}\right)$ correspond to a rotation period of 3.5 days (1.6 days). Thus the variations in the $\mathrm{H}_{\alpha}$ and $\mathrm{H}_{\beta}$ flux seen from observation to observation (with a spacing of $\sim 1$ day) could be due to stellar rotation.

In addition the $\mathrm{H}_{\beta}$ flux is more affected by extinction than the $\mathrm{H}_{\alpha}$ flux. Thus the variations seen in the $\mathrm{H}_{\alpha}$ and $\mathrm{H}_{\beta}$ flux may be due to variations in the extinction. The ratio of the $\mathrm{H}_{\alpha}$ to $\mathrm{H}_{\beta}$ flux varies in the range $\sim 4.0-6.3$ (Table 3 ). To estimate the extinction (variations) we use the approach described in Scowen et al. (1992). The unreddened intensity $I_{\lambda}$ relative to the intensity of $\mathrm{H}_{\beta}\left(I_{\mathrm{H}_{\beta}}\right)$ is related to the measured intensity $F_{\lambda}$ relative to $F_{\mathrm{H}_{\beta}}$ as

$\frac{I_{\lambda}}{I_{\mathrm{H}_{\beta}}}=\frac{F_{\lambda}}{F_{\mathrm{H}_{\beta}}} 10^{C_{\mathrm{H}_{\beta}}}\left(f_{\lambda}-f_{\mathrm{H}_{\beta}}\right)$ 
with $f_{\lambda}-f_{\mathrm{H}_{\beta}}$ taken from the standard extinction curve. With $f(\mathrm{H} \alpha)-f\left(\mathrm{H}_{\beta}\right)=-0.323$ (Osterbrock 1974), one obtains with $\frac{I_{\mathrm{H}_{\alpha}}}{I_{\mathrm{H}_{\beta}}}=2.86$ (Osterbrock 1974)

$C_{\mathrm{H}_{\beta}}=3.096 \log \frac{F_{\mathrm{H}_{\alpha}}}{F_{\mathrm{H}_{\beta}}}-1.413$.

The optical depth $\tau_{\lambda}$ at the wavelength $\lambda$ is given by

$\tau_{\lambda}=C_{\mathrm{H}_{\beta}} f(\lambda)$

(Osterbrock 1974). The extinction in the $V$-band ( 15561$)$ is then determined with $f(\lambda 5561)=0.84$ as

$A_{\mathrm{V}}=-2.5 \log \left(\mathrm{e}^{-0.84 C_{\mathrm{H}_{\beta}}}\right)$.

The observed flux ratio $\mathrm{H}_{\alpha}$ to $\mathrm{H}_{\beta}$ gives with Eq. (4) a range in visual extinction $A_{\mathrm{V}}=0.42-0.96$. The equivalent hydrogen absorbing column density is $\sim(0.65-1.4) \times 10^{21} \mathrm{~cm}^{-2}$.

We do not sufficiently resolve the $\mathrm{H}_{\alpha}$ profile of LMCV2135 and cannot infer an inclination from the $\mathrm{H}_{\alpha}$ line. We note that a high inclination $\left(i>33^{\circ}\right)$ may show up as double emission and central absorption and classify the source as a shell star (Briot 1986). In the further discussion we will use a value for the inclination angle of $60^{\circ}$.

In $\mathrm{Be} / \mathrm{NS}$ binaries smaller $\mathrm{H}_{\alpha}$ equivalent widths (of 10-20 ̊) are observed which indicate for smaller Be disks. This is consistent with higher eccentricities of NS orbits around Be stars which allow the NS to come closer to the Be star during periastron passage. There are two suspected Be/NS X-ray binaries identified in the Small Magellanic Cloud which also show large $\mathrm{H}_{\alpha}$ equivalent widths, XTE J0052-723 (43.3 A, Laycock et al. 2003) and RX J0101.0-7206 (54.6 ̊, Edge \& Coe 2003). The line profile of the first source is double-peaked which indicates for a likely high inclination while the line profile of the second source indicates that the Be star is observed close to the axis of rotation.

The $\mathrm{H}_{\alpha}$ and $\mathrm{H}_{\beta}$ lines show variations in the radial velocity during the observations covering $\sim 7$ days (Table 3 ). The range of radial velocities is $\sim 430 \mathrm{~km} \mathrm{~s}^{-1}$ to $\sim 6 \mathrm{~km} \mathrm{~s}^{-1}$ for the $\mathrm{H}_{\alpha}$ line and $\sim 450 \mathrm{~km} \mathrm{~s}^{-1}$ to $\sim 20 \mathrm{~km} \mathrm{~s}^{-1}$ for the $\mathrm{H}_{\beta}$ line. As the systemic velocity of the LMC is $\sim 278 \mathrm{~km} \mathrm{~s}^{-1}$ these variations in the radial velocities are consistent with a radial velocity amplitude of $\sim 170-225 \mathrm{~km} \mathrm{~s}^{-1}$. If these radial velocity variations are due to a Keplerian motion, then constraints can be derived for a Keplerian period, the component masses and the inclination of the orbit. We note that the rotation velocity of the star and the Keplerian velocity of the disk at a few stellar radii are comparable. The poor sampling of a likely Keplerian orbit does not allow to derive reliable constraints. We only note that the observed radial velocity variations are consistent with a Keplerian orbit of $\sim 10$ days around a B0-3 star as expected from the disk of such a star. The observed variations would require that the $\mathrm{H}_{\alpha}$ and the $\mathrm{H}_{\beta}$ emission is not isotropic across the Be disk. This may be due to anisotropic filling of the Be disk or longitudinal oscillations. We note that a period of $\sim 10$ days is consistent with periods of days found from the photometric variability of the optical counterparts of several Be/X-ray binaries which are explained as low-amplitude quasi-periodic variations of the equatorial Be disk (e.g. Schmidtke \& Cowley 2005).

\subsection{Binarity}

We found quasi-periodicities of $510 \pm 20$ and $1040 \pm$ 70 days from an FFT applied to the MACHO light curve of
XMMU J052016.0-692505. But we cover only a few cycles and cannot judge on a periodic nature of these variabilities. We thus cannot judge whether one of these variabilities is related to the orbital period of the likely binary system or to the activity of the Be star and its disk. An orbital period of a few hundred days is predicted from population synthesis calculations of Be/WD systems (Raguzova 2001). If XMMU J052016.0-692505 is a $\mathrm{Be} / \mathrm{WD}$ binary system then the WD is expected to be massive (a mass of 0.9-1.0 $M_{\odot}$ is likely) in agreement with what would be required from the mass and evolutionary state of the Be star companion.

\subsection{Stable nuclear burning WD}

We argue that the super-soft spectrum and high X-ray luminosity, the long-term optical light curve which shows no strong outbursts and may indicate for a rather circular orbit, are consistent with a Be/WD X-ray binary. If XMMU J052016.0-692505 is a Be/WD binary with steady nuclear burning on the WD surface, then the accretion rate onto the WD has to be sufficiently large that steady nuclear burning can be sustained. For a WD mass in the range $\sim 0.7-1.2 M_{\odot}$ the steady nuclear burning rate is $\sim(1-4) \times 10^{-7} M_{\odot} \mathrm{yr}^{-1}$. For lower accretion rates a stable nuclear burning phase can occur following a nova outburst (post-nova). The existence of Be/WD binary systems has been predicted in large numbers mainly from population synthesis calculations (e.g., Pols et al. 1991; Raguzova 2001). Only few such systems have been proposed from the observations so far. In order to estimate if a steady nuclear burning WD can occur in a Be/WD binary system we estimate the accretion rate onto the WD. We approach this question by using the method described by Waters et al. (1988).

We use a stellar system with a Be star of radius $R_{*}$ and with an equatorial disk. For the radial dependence of the density of such a disk $\rho(r)$ the description of Waters et al. (1988) is used

$\rho(r)=\rho_{0} \times\left(\frac{r}{R_{*}}\right)^{-n}$

with $3 \leq n \leq 3.75$. The wind velocity in such a disk is described as

$v_{\mathrm{w}}(r)=v_{0} \times\left(\frac{r}{R_{*}}\right)^{m}$

with $m=n-2$. We use the relative velocity $v_{\text {rel }}=v_{\mathrm{w}}$ with the wind velocity $v_{\mathrm{w}}, \rho_{0}=10^{-11} \mathrm{~g} \mathrm{~cm}^{-3}, M_{\mathrm{x}}=0.9 M_{\odot}, v_{0}=$ $5 \mathrm{~km} \mathrm{~s}^{-1}$ and $x=\frac{r}{R_{*}}$. We then derive the accretion rate from

$\dot{M}_{\mathrm{acc}}=2.31 \times 10^{18} x^{-(n+4 m)}\left(2.5 \times 10^{11} x^{2 m}+v_{\mathrm{orb}}^{2}\right)^{1 / 2} \mathrm{~g} \mathrm{~s}^{-1}$.

Using a less steep density profile $(n=3.0)$ for the Be disk as may be more consistent with the extended Be disk observed in the optical spectrum of LMCV2135 (cf. van Kerkwijk et al. 1995, Fig. 6), we infer a higher accretion rate of $\dot{M}_{\text {acc }}<1.5 \times$ $10^{-5} M_{\odot} \mathrm{yr}^{-1}$. We note that our estimate of the accretion rate is very uncertain. Hydrodynamic calculations as have been performed for the Be/X-ray binary system 4U 0115+63 (Hayasaki $\&$ Okazaki 2004) would be required. But such calculations cannot yet be performed for LMCV2135 as a complete set of stellar and orbital parameters has so far not yet been determined.

Another requirement for a stable nuclear burning WD is that the WD has accreted the critical envelope mass of H-rich matter. This mass strongly depends on the mass of the WD 
and less strongly on the accretion rate onto the WD. For a $0.9-1.0 M_{\odot}$ WD the critical envelope mass is $\sim(5-7) \times 10^{-5} M_{\odot}$ (e.g. Kahabka \& van den Heuvel 2006). Assuming an effective accretion rate of $<1.5 \times 10^{-7} M_{\odot} \mathrm{yr}^{-1}$ (assuming that the maximum accretion rate is $<1.5 \times 10^{-5} M_{\odot} \mathrm{yr}^{-1}$ when a stellar disk has formed and the stellar disk is present only $1 \%$ of the time), it will take $>5 \times 10^{2} \mathrm{yr}$ to accrete the critical envelope mass. The evolutionary age of a star which reproduces the temperature and radius of the observed optical continuum is $\sim 0.25-4.3 \times 10^{7} \mathrm{yr}$. Thus there may be sufficient time for accumulating a few critical envelopes. In addition the formation of a $0.9-1.0 M_{\odot}$ WD from a progenitor star, which is shorter than the evolutionary age of the Be star, has to be taken into account. Thus it appears possible that the WD is in a state of stable nuclear burning.

As the X-ray spectrum of the nuclear burning WD is supersoft it will be absorbed by the Be disk. The Hydrogen absorbing column density of a Be disk can be estimated with the density, Eq. (5)

$N_{\mathrm{H}}=\frac{\rho_{0}}{m_{\mathrm{p}}} \times\left(\frac{r}{R_{*}}\right)^{-n} h(r) / \cos i$

with $h(r)$ the height of the disk, $i$ the inclination angle and $m_{\mathrm{p}}$ the proton mass. Using $r=5 R_{*}, R_{*}=5$ to $10 R_{\odot}, h(r)=\beta r$, $\beta=0.04$ (Hony 2000), $i=60^{\circ}$ and $n=3.0, \rho_{0}=10^{-11} \mathrm{~g} \mathrm{~cm}^{-3}$, one gets $N_{\mathrm{H}}=(1.3-6.7) \times 10^{22} \mathrm{~cm}^{-2}$.

Making use of the observed Hydrogen column density, we can infer the distance $r_{0}$ of the WD from the Be star.

$r_{0}=\left(\frac{N_{\mathrm{H} m_{\mathrm{p}} \cos i}}{\beta \rho_{0} R_{*}^{n}}\right)^{1 /(1-n)}$.

With $N_{\mathrm{H}}<6 \times 10^{21} \mathrm{~cm}^{-2}$ one obtains $r_{0}>5 R_{*}$. This would constrain the orbital period to $>12$ days.

\subsection{Evolutionary considerations}

The evolutionary state of the likely WD Be/X-ray binary XMMU J052016.0-692505 can be constrained if we know the mass of the WD, the mass of the Be star, and the age of the system. Assuming the super-soft flux distribution arises from the WD surface and using temperature - luminosity tracks for steady nuclear burning WDs (Iben 1982; Nomoto et al. 2006) we constrain the WD mass to $\sim 0.87-0.97 M_{\odot}(90 \%$ confidence) by comparing with the allowed luminosity range from Fig. 2. Using WD (non-LTE and LTE) model atmospheres may somewhat change the constraints for the WD mass. Also for a cooling WD a higher WD mass is consistent with the spectral parameters. From the spectral flux distribution in the optical we constrain the optical star to a $\sim 7-15 M_{\odot}$ star with an age of 2.5-43 Myr using stellar evolutionary tracks. Thus the spectral type of the star would be B0-3 and the presence of strong $\mathrm{H}_{\alpha}$ and $\mathrm{H}_{\beta}$ emission lines in the optical spectrum would classify the optical star as B0-3e. A WD Be/X-ray binary with a mass of $\sim 0.9-1.0 M_{\odot}$ and a Be star of mass $\sim 7-15 M_{\odot}$ would be consistent with the stellar evolutionary scenario of Raguzova (2001). A primary of initial mass 5.6-14 $M_{\odot}$ evolves to a He star which will fill its Roche lobe and transfer mass to a secondary (B star) of a lower mass. Mass is transferred from the He star to the B star during case $\mathrm{BB}$ evolution and the mass of the secondary will grow. The He star evolves to a WD of mass $\sim 0.9-1.4 M_{\odot}$. This scenario allows that the mass of the secondary (Be star) after the evolution of the primary to a WD is larger then the mass of the progenitor of the WD as some mass is transferred from the WD progenitor to the Be star. This may be consistent with the high rotation velocity derived from the $\mathrm{H}_{\alpha}$ full width. This scenario is also consistent with the result of a photometric survey of $\mathrm{Be}$ stars in 55 open clusters in the southern sky where $73 \%$ of the Be stars have been found to be most likely spun-up by binary mass transfer (Mc Swain et al. 2005). This scenario offers the possibility that binary super-soft X-ray sources are observed in a relatively young $\left(\sim 4 \times 10^{7} \mathrm{yr}\right)$ stellar population. Apparently such a population is required to explain super-soft sources found in a few external galaxies close to spiral arms and/or HII regions (cf. discussion in Kahabka 2005).

\section{Conclusions}

We have discovered a super-soft X-ray source XMMU J052016.0-692505 in the LMC which is optically identified with the LMC variable star LMCV2135, a $M A C H O$ and $O G L E$ variable. The EPIC-pn and MOS spectra are fitted with a blackbody spectrum with a bolometric luminosity $\gtrsim 10^{34} \mathrm{erg} \mathrm{s}^{-1}$. Assuming the bolometric luminosity does not exceed $10^{38} \mathrm{erg} \mathrm{s}^{-1}$, we infer a temperature of (25-70) $\mathrm{eV}$, consistent with stable nuclear burning on a $\sim 0.9-1.0 \quad M_{\odot}$ WD. The optical spectra are consistent with a Be star of spectral type B0-3e. They show strong $\mathrm{H}_{\alpha}$ and $\mathrm{H}_{\beta}$ emission lines indicating the presence of an extended $\mathrm{Be}$ disk. XMMU J052016.0-692505 thus may be a stable nuclear burning WD accreting from the disk of a Be star.

Acknowledgements. The XMM-Newton project is supported by the Bundesministerium für Wirtschaft und Technologie/Deutsches Zentrum für Luft- und Raumfahrt (BMWI/DLR, FKZ 50 OX 0001), the Max-Planck Society and the Heidenhain-Stiftung. This research has made use of the VizieR catalog access tool, CDS, Strasbourg, France. This paper utilizes public domain data originally obtained by the $M A C H O$ Project, whose work was performed under the joint auspices of the U.S. Department of Energy, National Nuclear Security Administration by the University of California, Lawrence Livermore National Laboratory under contract No. W-7405-Eng-48, the National Science Foundation through the Center for Particle Astrophysics of the University of California under cooperative agreement AST-8809616, and the Mount Stromlo and Siding Spring Observatory, part of the Australian National University. The optical observations at the SAAO facility is supported by the Australian Government. We thank the staff at the SAAO for their hospitality. Access to Major Research Facilities Program (AMRFP) grant - (05/06-o-11). We thank Dr. J. Greiner for helpful discussions. We thank the referee for helpful comments.

\section{References}

Anders, E., \& Grevesse, N. 1989, GeCoA, 53, 197

Aschenbach, B., Briel, U. G., Haberl, F., et al. 2000, in Proc. SPIE, X-ray Optics, Instruments, and Mirrors III, ed. J. E. Trümper, \& B. Aschenbach, 4012, 731 Briot, D. 1986, A\&A, 163, 67

Brüns, C., Kerp, J., Staveley-Smith, L., et al. 2005, A\&A, 432, 937

Cash, W. 1979, ApJ, 228, 939

Dachs, J., Hanuschik, R., Kaiser, D., \& Rohe, D. 1986, A\&A, 159, 276

Edge, W. R. T., \& Coe, M. J. 2003, MNRAS, 338, 428

Hayasaki, K., \& Okazaki, A. T. 2004, MNRAS, 350, 971

Harmanec, P. 1988, BAICz, 39, 329

Hony, S., Waters, L. B. F. M., Zaal, P. A., et al. 2000, A\&A, 355, 187

Iben, I. Jr 1982, ApJ, 259, 244

Kahabka, P. 2005, Supersoft X-ray sources, in COSPAR Colloquium Spectra \&

Timing of Compact X-ray Binaries, ed. P. Ghosh, \& E. P. J. van den Heuvel Kahabka, P., \& Haberl, F. 2006, A\&A, 452, 431

Kahabka, P., \& van den Heuvel, E. P. J. 2006, in Compact Stellar X-Ray Sources, ed. W. H. G. Lewin, \& M. van der Klis, Cambridge University Ser., 461 Laycock, S., Corbet, R. H. D., \& Coe, M. J. 2001, MNRAS, 339, 435 Lejeune, Th., Cuisinier, F., \& Buser, R. 1997, A\&AS, 125, 229

Lomb, N. R. 1976, Ap\&SS, 39, 447

Mason, K. O., Breeveld, A., Much, R., et al. 2001, A\&A, 365, L36

Massey, P. 2002, ApJS, 141, 81

McSwain, M. V., \& Gies, D. R. 2005, ApJS, 161, 118 
Mennickent, R. E., Pietrzyński, G., Gieren, W., \& Szewczyk, O. 2002, A\&A, 393,887

Nomoto K., Saio, H., Kato, M., \& Hachisu, I. 2006, ApJ, submitted [arXiv:astro-ph/0603351]

Oliveira, R. L., Motch, C., Haberl, F., et al. 2006 [arXiv: astro-ph/0603098] Osterbrock, D. E. 1974, Astrophysics of Gaseous Nebulae (San Francisco: W. H. Freeman and Company)

Pols, O. R., Cote, J., Waters, L. B. F. M., \& Heise, J. 1991, A\&A, 241, 419

Raguzova, N. V. 2001, A\&A, 367, 848

Samus, N. N., Durlevich, O. V., et al. 2004, VizieR On-line Data Catalog: II/250 (GCVS4.2)

Scargle, J. D. 1982, ApJ, 263, 835

Scowen, P. A., Dufour, R. J., \& Hester, J. J. 1992, AJ, 104, 92
Schmidtke, P. C., \& Cowley, A. P. 2005, AJ, 130, 2220

Starrfield, S., Timmes, F. X., Hix, W. R., et al. 2004, ApJ, 612, L53

Strüder, L., Briel, U., Dennerl, K., et al. 2001, A\&A, 365, L18

Turner, M. J. L., Abbey, A., Arnaud, M., et al. 2001, A\&A, 365, L27

Udalski, A., Kubiak, M., \& Szymański, M. 1997, Acta Astron., 47, 319

Udalski, A., Szymański, M., Kubiak, M., et al. 1998, Acta Astron., 48, 147

van den Heuvel, E. P. J., Bhattacharya, D., Nomoto, K., \& Rapppaport, S. A. 1992, A\&A, 262, 97

van Kerkwijk, M. H., Waters, L. B. F. M., \& Marlborough, J. M. 1995, A\&A, 300,259

Waters, L. B. F. M., Taylor, A. R., van den Heuvel, E. P. J., et al. 1988, A\&A, 198,200 